\title{
Editorial: Behavioral Adaptations to Life in the City
}

\author{
David Luther ${ }^{1 *}$ and Elizabeth P. Derrberry ${ }^{2}$ \\ ${ }^{1}$ Department of Biology, George Mason University, Fairfax, VA, United States, ${ }^{2}$ Department of Ecology and Evolutionary \\ Biology, University of Tennessee, Knoxville, TN, United States
}

Keywords: urban, phenotype, environment, conservation, ecology

\section{Editorial on the Research Topic}

\section{Behavioral Adaptations to Life in the City}

Humans have drastically transformed the planet and recent estimates indicate that only $3 \%$ of terrestrial ecosystems remain ecologically intact (Plumptre et al., 2021). A fundamental challenge of ecologists, evolutionary biologists, and conservation practitioners is to understand how animals are responding to these rapid ecosystem changes and novel environments. Urban regions are among the newest and most rapidly expanding habitats as well as the most altered terrestrial ecosystems on the planet (Grimm et al., 2008; Seto et al., 2012; Rivkin et al., 2019). Studies that focus on urban phenotypes and seek to understand what traits permit animals to permeate the urban filter, have provided a greater understanding of which animals and functional groups are best able to adapt to novel urban environments. In addition, population-level studies that focus on changes in physiology, morphology, and behavior have elucidated the manners in which animals are most likely to adapt and survive in urban areas. However, it is not entirely clear what aspects of urban living are responsible for urban phenotypes or if urban adaptations lead to meaningful divergence from rural populations.

Urban environments present novel abiotic and biotic environments for species. Novel abiotic

Edited and reviewed by: Elise Huchard,

UMR5554 Institut des Sciences de I'Evolution de Montpellier (ISEM), France

*Correspondence: David Luther dluther@gmu.edu

Specialty section:

This article was submitted to Behavioral and Evolutionary Ecology, a section of the journal

Frontiers in Ecology and Evolution

Received: 29 May 2021

Accepted: 21 June 2021

Published: 13 July 2021

Citation: Luther D and Derrberry EP (2021) Editorial: Behavioral Adaptations to Life in the City.

Front. Ecol. Evol. 9:716713. doi: 10.3389/fevo.2021.716713 changes include anthropogenic noise, artificial light, heat islands, and chemical pollution, all of which strongly influence animal behavior, physiology, and population density (Swaddle et al., 2015; Sparkman et al., 2018; Putman et al., 2019). Novel biotic changes include shifted diets and foraging opportunities, depauperate faunal communities, heightened levels of non-native species and altered predator communities (Isaksson and Andersson, 2007; Narango et al., 2017; Seress et al., 2018). Understanding how animals respond and adapt to these novel environmental factors can help us understand which species are able to adapt to urban environments, and how urban environments might be altered to accommodate more native species.

This special issue on Behavioral Adaptations to Life in the City brings together a collection of articles to explore fundamental questions that to date have been unanswered. The goal of the special issue is to explore these questions, fill in knowledge gaps regarding urban phenotypes and present the latest theoretical and empirical research on behavioral flexibility and adaptations to urban environments. With the recent pandemic came increased awareness of animal life in cities, and these studies address the recent surge in interest as to how animals succeed or fail in urbanized habitats. The special issue identifies traits of the urban phenotype and discusses potential fitness consequences of those traits.

Several papers in this issue confirm and expand upon many specific traits, such as bird song and nesting behavior, that have been observed or hypothesized to be typical of the urban phenotype. For example Variation in Diurnal Patterns of Singing Activity Between Urban and Rural Great Tits by Bermúdez-Cuamatzin et al., find differences in singing activity of great tits between urban and rural areas such that urban tits sing earlier during the day, 
with similar singing effort during the dawn chorus and lower singing effort later during the day as compared to rural birds. Temporally Separated Data Sets Reveal Similar Traits of Birds Persisting in a United States Megacity by Cooper et al. analyzes life history and behavioral traits associated with urbanization for 52 breeding bird species on 173 survey blocks in the Los Angeles area of southern California, United States, in the late 1990s and again in the 2010s. This study finds that cavity-nesting and migratory behavior are filters that prevent successful persistence in urban areas as they are negatively associated with urbanization, and nesting on human-made structures is positively associated with urbanization. Winandy et al. assess how urban noise restricts song frequency bandwidth and syllable diversity in bananaquits: increasing audibility at the expense of signal quality and imply negative consequences in terms of sexual selection for reduced signal quality. Winandy et al. Noise-Related Song Variation Affects Communication: Bananaquits Adjust Vocally to Playback of Elaborate or Simple Songs nicely complements their other paper with playback experiments to reveal that songs with reduced syllable diversity and bandwidth receive weaker responses from territorial rivals, thus have an associated cost in terms of sexual selection. Phillips et al. find evidence that Territory Quality Predicts Avian Vocal Performance Across an Urban-Rural Gradient. The pattern of association between vocal performance, vegetation characteristics, and ambient noise levels, provides intriguing insights and raises new questions about the underlying mechanisms driving variation in song performance. In their paper Incubation Behavior Differences in Urban and Rural House Wrens, Troglodytes aedon Heppner and Ouyang find that urban females spend less time incubating, and have more and shorter incubation bouts, in association with higher ambient temperatures than rural females, which could be a consequence of the urban heat island effect.

While the aforementioned papers explore previously known urban phenotypes, several papers in this issue investigate which aspects of the urban environment organize urban communities and their spatial orientation within cities. Vigilance Response of a Key Prey Species to Anthropogenic and Natural Threats in Detroit by Lima et al. find that urban rabbits are most vigilant in areas with high domestic dog activity and that they avoid areas with carnivores such as coyotes. Meanwhile Hansen et al. explore the fundamental ecological relationship of habitat productivity and species distributions but do so within the confines of an urban area. Does Use of Backyard Resources Explain the Abundance of Urban Wildlife quantifies the relative influence of anthropogenic resources (food, water, shelter) in residential yards on the relative abundance of mammals. They find that food resources are an attractant of mammals to yards and predator species are associated

\section{REFERENCES}

Grimm, N. B., Faeth, S. H., Golubiewski, N. E., Redman, C. L., Wu, J., Bai, X., et al. (2008). Global change and the ecology of cities. Science 319, 756-760. doi: 10.1126/science.115 0195 with the relative abundance of mammalian prey species in those yards.

Finally, a few papers in this issue take a step back for a larger view of how animals are adapting to urban habitats with systematic reviews of the adaptations of mammals and of lizards to life in the city. Behavior Change in Urban Mammals: A Systematic Review by Ritzel and Gallo present a systematic review of literature regarding urban mammal behavior over the last 50 years. They identify taxonomic, regional, and behavioral biases in the literature and discuss the adaptive responses (regulatory, acclimatory, and developmental) of mammals to urbanization. Putman and Tippie's Big City Living: A Global Meta-Analysis Reveals Positive Impact of Urbanization on Body Size in Lizards provides a systematic review of the different selective pressures in urban environments compared to natural environments and how they lead to changes in animal behavior, physiology, and morphology among lizards. Lizards living in urban environments are larger than rural lizards and urban lizards use larger perches and have longer hind limbs. In the theme of stepping back and seeing the bigger picture of the urban phenotype, Halfwerk presents an opinion piece entitled How Should We Study Urban Speciation, in which he provides a candid perspective that illustrates our current lack evidence to fully assess speciation in urban environments. Lailvaux also takes a big picture approach to the urban phenotype by reviewing the different selective pressures on urban as compared to rural green Anole lizards and using long-term mark recapture experiments to assess sex-ratios and population density in urban environments. Lailvaux's It's Not Easy Being Green: Behavior, Morphology, and Population Structure in Urban and Natural Populations of Green Anole (Anolis carolinensis) Lizards finds that urban and rural lizards have diverged significantly in terms of their behavior and morphology.

While this special issue shines light on the urban phenotype, and several of the environmental mechanisms that lead to their divergence from rural populations, many questions remain unanswered. The largest of which is that we don't know the extent to which divergence among urban and rural populations leads to fitness consequences, or even to genotypic changes. This issue advances our understanding of the urban phenotype while also highlighting the continued need for research on whether urban adaptations have led to meaningful divergence from rural populations.

\section{AUTHOR CONTRIBUTIONS}

DL and ED conceived, wrote, edited, and approved of the submission of this editorial article. All authors contributed to the article and approved the submitted version. 
Plumptre, A. J., Baisero, D., Belote, R. T., Vázquez-Domínguez, E., Faurby, S., et al. (2021). Where might we find ecologically intact communities? Front. For. Glob. Change 4:26. doi: 10.3389/ffgc.2021.626635

Putman, B. J., Gasca, M., Blumstein, D. T., and Pauly, G. B. (2019). Downsizing for downtown: limb lengths, toe lengths, and scale counts decrease with urbanization in western fence lizards (Sceloporus occidentalis). Urban Ecosyst. 22, 1071-1081. doi: 10.1007/s11252-019-00889-Z

Rivkin, L. R., Santangelo, J. S., Alberti, M., Aronson, M. F., de Keyzer, C. W., Diamond, S. E., et al. (2019). A roadmap for urban evolutionary ecology. Evolut. Appl. 12, 384-398. doi: 10.1111/eva.12734

Seress, G., Hammer, T., Bókony, V., Vincze, E., Preiszner, B., Pipoly, I., et al. (2018). Impact of urbanization on abundance and phenology of caterpillars and consequences for breeding in an insectivorous bird. Ecol. Appl. 28, 1143-1156. doi: 10.1002/eap.1730

Seto, K. C., Güneralp, B., and Hutyra, L. R. (2012). Global forecasts of urban expansion to 2030 and direct impacts on biodiversity and carbon pools. Proc. Natl. Acad. Sci. U.S.A. 109, 16083-16088. doi: 10.1073/pnas.121165 8109
Sparkman, A., Howe, S., Hynes, S., Hobbs, B., and Handal, K. (2018). Parallel behavioral and morphological divergence in fence lizards on two college campuses. PLoS ONE 13:e0191800. doi: 10.1371/journal.pone.0191800

Swaddle, J. P., Francis, C. D., Barber, J. R., Cooper, C. B., Kyba, C. C. M., Dominoni, D. M., et al. (2015). A framework to assess evolutionary responses to anthropogenic light and sound. Trends Ecol. Evolut. 30, 550-560. doi: $10.1016 /$ j.tree.2015.06.009

Conflict of Interest: The authors declare that the research was conducted in the absence of any commercial or financial relationships that could be construed as a potential conflict of interest.

Copyright (c) 2021 Luther and Derrberry. This is an open-access article distributed under the terms of the Creative Commons Attribution License (CC BY). The use, distribution or reproduction in other forums is permitted, provided the original author(s) and the copyright owner(s) are credited and that the original publication in this journal is cited, in accordance with accepted academic practice. No use, distribution or reproduction is permitted which does not comply with these terms. 